\title{
Transition from Au to pseudo-Ga catalyzed growth mode observed in GaAs nanowires grown by molecular beam epitaxy
}

\author{
M. Soda, A. Rudolph, D. Schuh, J. Zweck, D. Bougeard, and E. Reiger \\ Institute for Experimental and Applied Physics, University of Regensburg, Universitätsstr. 31, D-93053 Regensburg, Germany
}

(Received 7 September 2011; revised manuscript received 5 April 2012; published 29 June 2012)

\begin{abstract}
We investigate the correlation between the Ga concentration of the catalyst droplet and the adopted crystal structure of individual GaAs nanowires grown by molecular-beam epitaxy using $\mathrm{Au}$ as a catalyst material. Through a postgrowth analysis the Ga content of the catalyst droplet during growth is estimated and related to the observed crystal structure of the nanowires. Depending on the Ga concentration, we observe a transition from typical Au catalyzed to pseudo-Ga assisted nanowire growth: Nanowires with low Ga concentration of the catalyst droplet during growth form predominantly wurtzite crystal structures. For Ga concentrations higher than 75 at. \%, which we refer to as the pseudo-Ga assisted growth mode, the probability to form zinc-blende segments is strongly enhanced owing to the reduced droplet surface energy of the catalyst.
\end{abstract}

DOI: 10.1103/PhysRevB.85.245450

PACS number(s): 61.46.Km, 68.37.Lp, 81.05.Ea, 81.07.Gf

\section{INTRODUCTION}

To overcome the problems which originate from the planar fabrication technique of conventional electronic devices, e.g., increasing the transistor density in integrated circuits, vertical nanowire structures are discussed as possible building blocks for future applications. Semiconducting nanowires already showed their potential for nanoscale electronics, ${ }^{1-4}$ photonics, ${ }^{5-7}$ and biological analysis. ${ }^{8}$ One peculiarity of epitaxial nanowire growth is that nanowires do not necessarily adopt the crystal structure of the substrate material. In the case of GaAs nanowires zinc-blende (ZB) as well as wurtzite (WZ) crystal structures can be formed. Defects like stacking faults and crystal phase mixing are often observed within single nanowires ${ }^{9}$ which may lower the performance of nanowirebased devices. Therefore, to fully exploit the potential of nanowires, control over the nanowire crystal structure is essential.

Semiconducting nanowires can be fabricated epitaxially on semiconductor substrates in a bottom up approach with the help of a catalyst. The catalyst, which initiates the nanowire growth, can be from the group-III element of a compound semiconductor, e.g., Ga in the case of GaAs nanowires, ${ }^{10}$ or from a different material such as metals. ${ }^{11}$ For Au catalyzed nanowire growth the Au forms an eutectic alloy with the semiconductor material when the substrate temperature exceeds a critical value. ${ }^{12}$ Liquid catalyst droplets are formed whose diameter sets the diameter of the nanowire. Because of the different aggregate states involved in nanowire growth, the growth model is referred to as a vapor-liquid-solid growth mechanism.

Starting nanowire growth with a thin Au film as a catalyst material typically generates a nanowire ensemble with a large variance in nanowire length and diameter. ${ }^{13}$ This is caused by small differences in the Au film thickness, surface roughening, or surface preparation leading to the formation of $\mathrm{Au}-\mathrm{Ga}$ droplets which are unequal in size and to locally varying catalyst droplet densities. ${ }^{14}$ The nanowire growth is a diffusion driven process: At the early stage $\mathrm{Ga}$ adatom diffusion takes place mainly at the substrate whereas at a later stage diffusion on the side facets becomes more and more important. ${ }^{15}$ As a consequence the global growth parameters such as applied material fluxes can be significantly modified for individual nanowires depending on the local surrounding of the nanowires. Nanowires originating from the same sample may thus experience different local growth conditions and show different crystal structures.

In our study, we investigate the correlation between the crystal structure and the chemical composition of the catalyst droplet during growth for individual nanowires. From a postgrowth transmission electron microscopy (TEM) analysis the $\mathrm{Ga}$ concentration of individual catalyst droplets during growth is estimated. We relate this parameter to the observed crystal structure of the corresponding nanowires. For high Ga concentrations of the catalyst droplet our results indicate that the nanowire growth resembles more the $\mathrm{Ga}$ assisted than the Au catalyzed growth mode. As a consequence the formation of zinc-blende segments becomes more probable. We discuss our findings within the nucleation-based growth model for $\mathrm{Au}$ catalyzed nanowire growth. ${ }^{16}$

\section{EXPERIMENT}

The GaAs nanowire samples were grown by molecularbeam epitaxy (MBE) using (111)B oriented GaAs substrates. The MBE - a modified Veeco Gen II system-is equipped with a reflection high-energy electron-diffraction (RHEED) system, a pyrometer specified for high temperatures, and an ion gauge. The $\mathrm{As}_{4}$ flux is given by the beam equivalent pressure (BEP) measured with the ion gauge. The Ga flux is typically determined by monitoring RHEED oscillations on a (001) oriented RHEED sample. Using a calibration function which was acquired by measuring the Ga flux and the Ga BEP for different Ga cell temperatures, the Ga flux can be also specified as the BEP value. The $\mathrm{As}_{4} / \mathrm{Ga}$ flux ratio can then be calculated from the BEP of both beams taking into account the absolute temperatures, the molecular weight, and the ionization efficiency relative to nitrogen of the two specimens. ${ }^{17}$

For sample preparation a thin Au film (1.5 or $10 \AA)$ was evaporated onto a GaAs wafer in a separate metal MBE system. 
TABLE I. Growth parameters and Au layer thickness: for catalyst preparation all samples were covered with a thin Au layer; samples A-D are grown with typical growth parameters for $\mathrm{Au}$ catalyzed nanowire growth; for sample $\mathrm{E}$ a comparatively small $\mathrm{As}_{4} / \mathrm{Ga}$ flux ratio was chosen.

\begin{tabular}{lccccc}
\hline \hline Sample & $\begin{array}{c}\text { Au layer } \\
(\AA)\end{array}$ & $\begin{array}{c}\mathrm{A}_{\mathrm{s} 4} \text { flux } \\
\left(10^{-6} \text { Torr }\right)\end{array}$ & $\begin{array}{c}\text { Ga rate } \\
(\AA / \mathrm{s})\end{array}$ & $\begin{array}{c}\mathrm{A}_{\mathrm{s} 4} / \mathrm{Ga} \\
\text { ratio }\end{array}$ & $\begin{array}{c}\text { Temp. } \\
\left({ }^{\circ} \mathrm{C}\right)\end{array}$ \\
\hline $\mathrm{A}$ & 10 & 1.28 & 0.38 & 3.90 & 534 \\
$\mathrm{~B}$ & 10 & 2.07 & 1.13 & 2.20 & 540 \\
$\mathrm{C}$ & 10 & 1.22 & 0.85 & 1.70 & 534 \\
$\mathrm{D}$ & 1.5 & 1.21 & 0.80 & 1.77 & 539 \\
$\mathrm{E}$ & 10 & 0.65 & 1.06 & 0.70 & 543 \\
\hline \hline
\end{tabular}

After chemical cleaning and heating at $350{ }^{\circ} \mathrm{C}$ for at least $1 \mathrm{~h}$ in a buffer chamber, the substrate was transferred to the growth chamber and set to $580{ }^{\circ} \mathrm{C}$ for $30 \mathrm{~min}$ to remove the natural oxide of GaAs and to form Au-Ga catalyst droplets. The oxide desorption was monitored by RHEED. During growth the substrate temperature was set to approximately $540^{\circ} \mathrm{C}$ for all samples and the substrate was rotated by seven rounds per minute. For growth termination the Ga cell was closed and the substrate heating was switched off. The As cell was kept open for an additional $5 \mathrm{~min}$ until the substrate temperature decreased below $400^{\circ} \mathrm{C}$. For all nanowire samples the same growth termination procedure was applied.

We investigate five nanowire samples which mainly differ in the chosen $\mathrm{Ga}$ and $\mathrm{As}_{4}$ fluxes as described in Table I. For samples A-D typical growth conditions for Au catalyzed nanowires were chosen with material fluxes in the range of $0.38-1.13 \AA / \mathrm{s}$ for $\mathrm{Ga}$ and $1.21 \times 10^{-6}-2.07 \times 10^{-6}$ Torr for $\mathrm{As}_{4}$, yielding $\mathrm{As}_{4} / \mathrm{Ga}$ ratios between 1.70 and 3.90. ${ }^{18,19}$ Sample $\mathrm{E}$ is grown under $\mathrm{Ga}$ rich growth conditions with a very small $\mathrm{As}_{4} / \mathrm{Ga}$ flux ratio of 0.70 .

After growth termination the nanowires and in particular the catalyst droplets at the tip of the nanowires were investigated by TEM. The nanowires were stripped off the substrate onto a holey carbon film TEM grid. Energy dispersive $\mathrm{X}$-ray spectroscopy (EDX) measurements and transmission electron microscopy (TEM) were carried out to investigate the chemical composition and the size of the catalyst. The crystal structure of the nanowires was analyzed using TEM electron-diffraction and high-resolution transmission electron microscopy (HRTEM) images.

\section{TEM ANALYSIS OF THE CATALYST DROPLET}

In a TEM postgrowth analysis we observe that the catalyst droplets and the tips of the nanowires can have significantly different shapes, even for nanowires originating from the same sample. In Fig. 1 TEM images of the tips of four different nanowires are depicted originating from sample A [Figs. 1(a) and 1(b)], sample D [Fig. 1(c)], and sample E [Fig. 1(d)]. The catalyst droplet diameter either equals approximately the diameter of the nanowire or is smaller in size. Only for very $\mathrm{Ga}$ rich growth conditions (sample E) a phase separation during growth termination can be observed leaving a small Au-Ga droplet within a large Ga droplet [Fig. 1(d)].

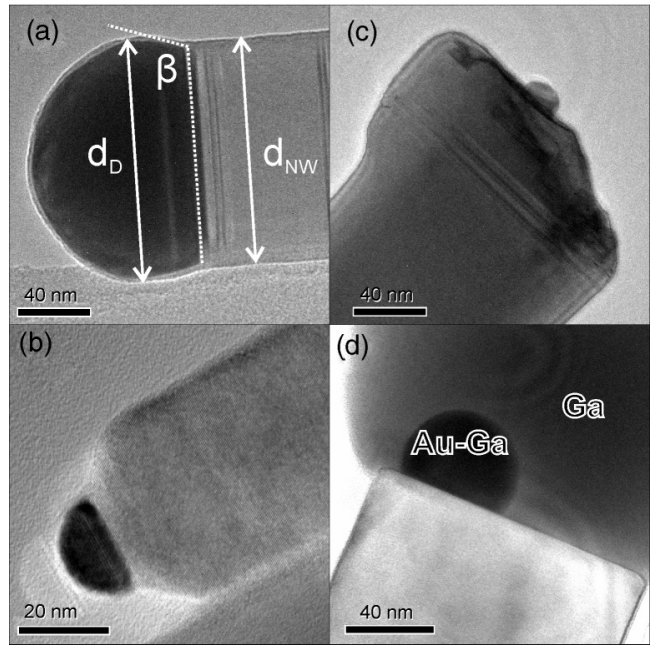

FIG. 1. TEM images of the nanowire tip. Different forms are observed: the diameter of the postgrowth catalyst droplet, $d_{D}$, either (a) equals the diameter of the nanowire, $d_{\mathrm{NW}}$, or (b and c) is considerably smaller in size. (d) For very Ga rich growth conditions a phase separation within the catalyst droplet can be observed. The nanowires shown in (a) and (b) are from sample A, whereas the nanowires in (c) and (d) are from samples D and E, respectively.

It is commonly accepted that during growth the size of the catalyst droplet equals - at least - the size of the nanowire. ${ }^{20,21}$ We attribute the reduced size of the postgrowth catalyst droplet to a partial incorporation of $\mathrm{Ga}$ dissolved in the catalyst droplet into the nanowire crystal during growth termination. ${ }^{11}$ Since for all samples the $\mathrm{As}_{4}$ cell is kept open until the substrate temperature cools down below $400{ }^{\circ} \mathrm{C}$ and since $\mathrm{Ga}$ is present in the catalyst droplet as well as on the side facets of the nanowire, nanowire growth can continue until the $\mathrm{As}_{4}$ supply is stopped or the substrate temperature decreases below a critical value. EDX measurements (not shown) prove that only $\mathrm{Ga}$ is incorporated, as no measurable amount of $\mathrm{Au}$ is detected in the nanowire close to the catalyst droplet. Due to the shrinking catalyst volume during growth termination, the nanowires exhibit a characteristic tapered shape [see Fig. 1(b)]. In literature this specific form of the tip of the nanowire is often referred to as a cooling neck. ${ }^{18,22}$

To quantitatively describe how strongly this cooling neck is developed, we introduce the nanowire-droplet (NWD) ratio, defined as

$$
\mathrm{NWD} \text { ratio }=\left(d_{\mathrm{NW}}-d_{D}\right) / d_{\mathrm{NW}},
$$

with $d_{\mathrm{NW}}$ and $d_{D}$ as the diameter of the nanowire and of the postgrowth catalyst droplet, respectively. In Fig. 1(a) the nanowire diameter $d_{\mathrm{NW}}$ as well as the postgrowth catalyst diameter $d_{D}$ and the postgrowth contact angle $\beta$ are sketched. As here the contact angle is slightly larger than $90^{\circ}$, the NWD ratio of -0.07 is negative. The nanowires shown in Figs. 1(b) and 1(c) exhibit NWD ratios of 0.48 and 0.84 , respectively.

With EDX we study the chemical composition of the postgrowth catalyst droplets. In Fig. 2 the ratio of the $\mathrm{Ga}_{K_{\alpha}}$ and the $\mathrm{Au}_{L_{\alpha}}$ peak is plotted versus the NWD ratio for different nanowires originating from sample $\mathrm{A}$ and sample $\mathrm{C}$. Interestingly, the data points are distributed into two regions. Nanowires with a NWD ratio $<0.1$ exhibit a significantly 


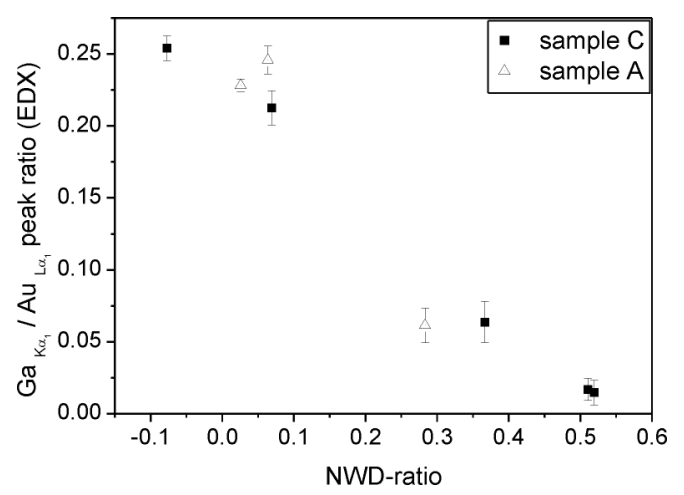

FIG. 2. EDX: Ga/Au peak ratio of the postgrowth catalyst droplet. Nanowires with a NWD ratio $\geqslant 0.3$ show a lower $\mathrm{Ga} / \mathrm{Au}$ peak ratio compared to nanowires with a NWD ratio $<0.1$.

higher $\mathrm{Ga} / \mathrm{Au}$ peak ratio than nanowires with a NWD ratio $\geqslant 0.3$. In other words, for nanowires which show a pronounced cooling neck [see Figs. 1(c) and 1(b)] the Ga concentration of the postgrowth catalyst is smaller than for nanowires whose catalyst droplet volume does not change significantly [see Fig. 1(a)]. From the correlation between the $\mathrm{Ga} / \mathrm{Au}$ peak and the NWD ratio we deduce the following: For nanowires with NWD $<0.1$ the postgrowth catalyst droplet contains approximately the same amount of $\mathrm{Ga}$ as during growth. In contrast, for nanowires with NWD $\geqslant 0.3$ the Ga concentration of the catalyst droplet was larger during growth than in the probed postgrowth analysis. The EDX measurements thus support the picture of the (partial) incorporation of $\mathrm{Ga}$ stemming from the catalyst droplet during growth termination. Note that the obtained values for the Au-Ga ratio by EDX are not an absolute measure because the element and transmission electron microscopy (TEM) specific Cliff-Lorimer factors, which are needed for the determination of the absolute concentration, are affected by large errors and were therefore not utilized. However, for our study the comparison of the uncorrected Au-Ga peak ratio is sufficient.

\section{RELATING THE CRYSTAL STRUCTURE TO THE NWD RATIO}

We investigated 22 nanowires originating from samples A-D (see Table I). The crystal structure of the nanowires was determined by analyzing TEM diffraction patterns. In Figs. 3(a) and 3(b) the diffraction pattern of two different

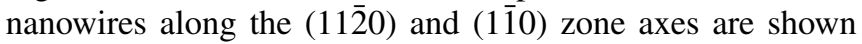
exemplarily. The diffraction patterns, characteristic for the wurtzite (WZ) [Fig. 3(a)] and zinc-blende (ZB) [Fig. 3(b)] crystal phases, respectively, can clearly be distinguished and are a definite proof for the appearance of a single-crystal phase in the probed part of the nanowire which is given by the electron spot size of $\approx 20 \mathrm{~nm}$. In contrast, the diffraction pattern shown in Fig. 3(c) stems from a nanowire with a mixed crystal structure. This type of diffraction pattern forms either due to a high density of single monolayer stacking faults or due to alternating segments of $\mathrm{ZB}$ and WZ crystal structure as shown in Fig. 3(d) and the inset, where short ZB segments are embedded in a WZ matrix.

All investigated nanowires were scanned taking TEM diffraction patterns along their entire length. The dominant

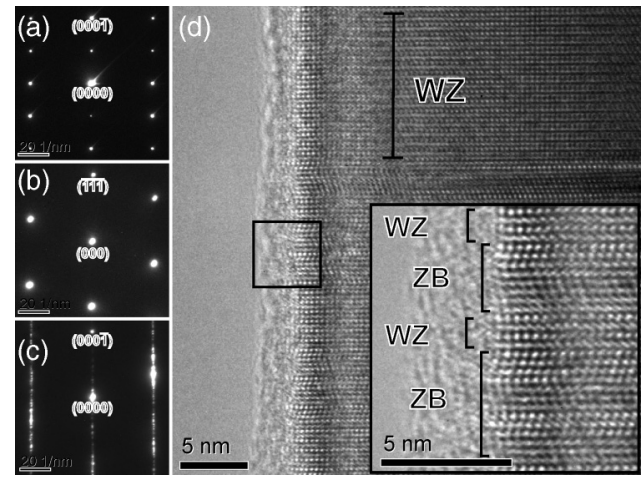

FIG. 3. TEM Diffraction patterns and HRTEM micrograph: diffraction pattern of (a) WZ (112̄0) zone axis, (b) ZB (11̄0) zone axis, and (c) nanowire region with an inexplicit crystal structure. (d) HRTEM micrograph of a nanowire region with WZ crystal structure and small ZB segments embedded.

crystal structure of all nanowires is the WZ crystal phase. The nanowires are divided in two classes, pure WZ nanowires (WZ) and nanowires which show both crystal structures $(\mathrm{WZ}+\mathrm{ZB})$. The nanowires are classified according to the observed diffraction patterns. Only if a mixed diffraction pattern was observed, HRTEM images were taken to clarify if a high density of stacking faults or if segments of $\mathrm{ZB}$ crystal structure are present. If the observed ZB segments consists of at least seven monolayers which correspond to a length of approximately $\geqslant 2 \mathrm{~nm}$, the nanowires are labeled as $\mathrm{WZ}+\mathrm{ZB}$. Otherwise they are assigned to $\mathrm{WZ}$ nanowires.

The results of our analysis are summarized in Fig. 4, which shows the number of $\mathrm{WZ}+\mathrm{ZB}$ or $\mathrm{WZ}$ nanowires with respect to their NWD ratio. Nanowires with a NWD ratio smaller than 0.2 crystallize in a pure $\mathrm{WZ}$ crystal structure. There exists an intermediate regime $(0.2 \leqslant \mathrm{NWD}$ ratio $\leqslant 0.4)$ where $\mathrm{WZ}$ as well as $\mathrm{WZ}+\mathrm{ZB}$ nanowires are observed. For NWD ratios higher than 0.4 all nanowires form $\mathrm{ZB}$ segments.

WZ nanowires show hexagonal or polygonal cross sections with $\{11 \overline{2} 0\}$ and $\{11 \overline{2} 0\}+\{1 \overline{1} 00\}$ oriented side facets, whereas $\mathrm{WZ}+\mathrm{ZB}$ nanowires have predominantly a hexagonal cross section with $\{11 \overline{2} 0\}$ oriented side facets.

\section{GA CONCENTRATION OF THE CATALYST DROPLET DURING GROWTH}

For the formation of a distinct crystal phase of the nanowires the essential parameter is the $\mathrm{Ga}$ concentration, $x_{\mathrm{Ga}}$, of the catalyst droplet during nanowire growth. As we will show the NWD ratio is linked to the Ga concentration of the catalyst droplet during growth. From the postgrowth EDX (see Fig. 2) and TEM analysis we estimate $x_{\mathrm{Ga}}$ as follows: For nanowires with NWD $<0.1$, as we cannot extract absolute Ga concentrations from the EDX measurements, we have to go back to values from literature. From the binary Au-Ga phase diagram a theoretical lower bound of $\approx 25$ at. $\%$ can be derived, as only for Ga concentrations higher than 25 at. \% the AuGa alloy is liquid at typical nanowire growth temperatures. ${ }^{23}$ Calculations of the Au-Ga-As ternary phase diagram close to $\mathrm{MBE}$ conditions (pressure $10^{-5} \mathrm{bar}$, temperature $590^{\circ} \mathrm{C}$ ) 

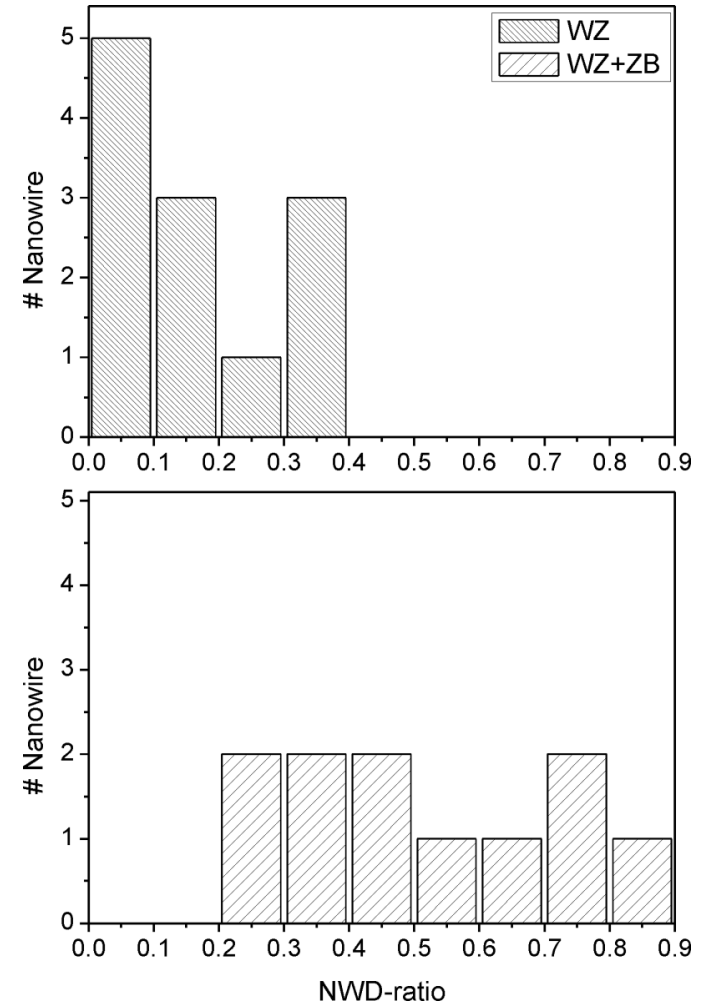

FIG. 4. Number of $\mathrm{WZ}$ and $\mathrm{WZ}+\mathrm{ZB}$ nanowires as a function of the NWD ratio: The observed crystal structure is clearly correlated with the NWD ratio. Nanowires with a NWD ratio smaller than 0.2 have a pure WZ crystal phase whereas all nanowires with a NWD ratio larger than 0.4 exhibit $\mathrm{ZB}$ segments embedded in a WZ crystal matrix.

show that the As concentration within the catalyst droplet is negligible with an As concentration lower than 0.2 at. \% for Ga concentration higher than 30 at. \% ${ }^{24}$ To our knowledge the only experimentally obtained Ga concentrations are reported by Harmand et al., who determined Ga concentrations of 0.37 and 0.50 of the catalyst droplet using an abrupt growth termination process. ${ }^{11}$

To obtain a lower bound of $x_{\mathrm{Ga}}$ for nanowires with NWD $\geqslant 0.3$ we use the following assumptions. The shape of the catalyst droplet is assumed to be hemispherical. The postgrowth catalyst droplet is supposed to have no $\mathrm{Ga}$ or As contributions. ${ }^{25}$ The number of Au atoms in the catalyst droplet can then be calculated from the volume of the postgrowth catalyst. During growth a contact angle of $90^{\circ}$ is considered; i.e., the volume of the AuGa catalyst during growth is determined by the nanowire diameter-giving a lower bound for the estimated $\mathrm{Ga}$ concentration. From the difference of both volumes the number of $\mathrm{Ga}$ atoms and hence the $\mathrm{Ga}$ concentration can be calculated.

In Fig. 5 the results of our estimate are summarized. For nanowires with NWD $<0.1$, values from literature are drawn as solid lines giving a lower and upper bound. For nanowires with NWD $>0.3$, the calculated minimum $x_{\mathrm{Ga}}$ value increases with increasing NWD ratio from approximately 60 at. $\%$ at NWD ratio 0.3 to values larger than 90 at. \% for NWD ratios larger than 0.6 .

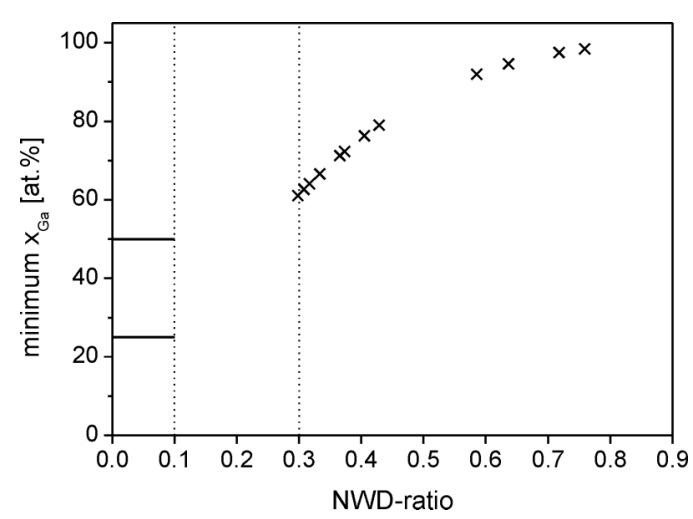

FIG. 5. Ga concentration of the catalyst droplet during growth: Solid lines are values taken from literature; crosses give a lower bound for the Ga concentration calculated for experimentally observed NWD ratios.

Comparing the estimated Ga concentrations (Fig. 5) with the observed crystal structure of the nanowires (Fig. 4) we conclude that a high $\mathrm{Ga}$ concentration of the catalyst droplet during nanowire growth favors the formation of ZB segments. Nanowires with a NWD ratio larger than 0.4 - which corresponds to a Ga concentration higher than 75 at. \%-exhibit ZB segments. Lower Ga concentrations lead to the formation of pure WZ nanowires with NWD ratios smaller than 0.2. In the intermediate NWD ratio regime we observe $\mathrm{WZ}$ as well as $\mathrm{WZ}+\mathrm{ZB}$ segments.

Our conclusion is supported by comparison of the different nanowire samples (see Table I) which are grown with different $\mathrm{As}_{4} / \mathrm{Ga}$ ratios. If we compare the data obtained within each nanowire sample, we observe the same trend. For sample A, grown with the highest $\mathrm{As}_{4} / \mathrm{Ga}$ ratio of 3.9 , only $\mathrm{WZ}$ nanowires are detected. Decreasing the $\mathrm{As}_{4} / \mathrm{Ga}$ ratio to more Ga rich growth conditions increases the probability to find $\mathrm{WZ}+\mathrm{ZB}$ nanowires. For sample B ( $\mathrm{As}_{4} / \mathrm{Ga}$ ratio: 2.20$)$ two out of four nanowires are $\mathrm{WZ}+\mathrm{ZB}$, and for sample $\mathrm{C}\left(\mathrm{As}_{4} / \mathrm{Ga}\right.$ ratio: 1.70$)$ two out of five nanowires are $\mathrm{WZ}+\mathrm{ZB}$. The very thin Au layer of $1.5 \AA$ of sample D (instead of $10 \AA$ for all other samples) leads very likely to an enhanced $\mathrm{Ga}$ concentration of the catalyst droplets during catalyst formation as well as during growth, resulting in the formation of only $\mathrm{WZ}+\mathrm{ZB}$ nanowires.

\section{COMPARISON WITH THE NUCLEATION THEORY}

Next, we discuss our results within the nucleation-based growth models for Au catalyzed nanowire growth developed by Glas et al. ${ }^{16}$ In this model the formation of the $\mathrm{WZ}$ and the ZB crystal phases depends on where the two-dimensional nucleus for the monolayer growth is formed at the nanowirecatalyst boundary. If the nucleus is formed at the center $(\mathrm{C})$ of the nanowire-catalyst boundary, i.e., it is entirely surrounded by the liquid catalyst, nucleation occurs preferentially in the $\mathrm{ZB}$ position. In contrast, if the nucleation starts at the triple phase boundary (TPB), i.e., the lateral surface of the nucleus is partly surrounded by vapor, wurtzite formation is favored for certain ranges of the relevant interface energies.

To determine where the nucleus is formed, the difference in formation enthalpy of the nucleus at different positions of the droplet-nanowire boundary, $\Delta \gamma$, has to be calculated. 
Depending on the sign of $\Delta \gamma$, the nucleation at the center (C) of the droplet-nanowire boundary or at the triple phase boundary (TPB) is favored. As stated by Glas et al. ${ }^{16}$ and Cirlin et al., ${ }^{26}$ the condition for nucleation at the TPB is given by

$$
\Delta \gamma=\gamma_{\mathrm{WV}}-\gamma_{\mathrm{SL}}-\gamma_{\mathrm{LV}} \sin \beta<0,
$$

where $\gamma_{\mathrm{WV}}$ is the WZ sidewall surface energy, $\gamma_{\mathrm{SL}}$ is the solidliquid surface energy, $\gamma_{\mathrm{LV}}$ is the liquid surface energy of the catalyst droplet, and $\beta$ is the contact angle of the droplet. A positive value of $\Delta \gamma$ indicates that nucleation occurs at the center (C).

We calculate $\Delta \gamma$ for two different sidewall orientations, $\{11 \overline{2} 0\}$ and $\{1 \overline{1} 00\}$. For the WZ sidewall surface energy $\gamma_{\mathrm{WV}}$ we use values taken from Ref. 27: $\gamma_{\mathrm{WV}}(\mathrm{WZ1} 100)=$ $1.30 \mathrm{~J} / \mathrm{m}^{2}$ and $\gamma_{\mathrm{WV}}(\mathrm{WZ} 11 \overline{2} 0)=1.54 \mathrm{~J} / \mathrm{m}^{2}$. The solid-liquid surface energy, $\gamma_{\mathrm{SL}}=0.59 \mathrm{~J} / \mathrm{m}^{2}$, is identical for the $\mathrm{ZB}$ and WZ nuclei due to the close atomic environments on the surface around the two types of nuclei. ${ }^{16,21,26}$ The liquid surface energy $\gamma_{\mathrm{LV}}$ of the catalyst droplet is a function of the Ga concentration. As in Ref. 26 we linearly interpolate $\gamma_{\mathrm{LV}}$ between the value for pure gold $\left(1.25 \mathrm{~J} / \mathrm{m}^{2}\right)$ and pure gallium $\left(0.6753 \mathrm{~J} / \mathrm{m}^{2}\right)$ at the given growth temperature of $540{ }^{\circ} \mathrm{C} .{ }^{28}$

The results of our calculation are shown in Fig. 6. The upper (lower) plot shows the dependence of $\Delta \gamma$ for the $\{1 \overline{1} 00\}$ $(\{11 \overline{2} 0\})$ side facets, respectively. Two different contact angles $\beta$ of 90 and $120^{\circ}$ are considered as the lower and upper bounds. As the surface energy of $\mathrm{Ga}$ is lower compared to $\mathrm{Au}$, the contact angle increases with the $\mathrm{Ga}$ concentration of the AuGa catalyst droplet [see Fig. 1(d)]. For nanowires with an estimated $\mathrm{Ga}$ concentration between 25 and 50 at. $\%$ the contact angle is very likely close to $90^{\circ}$. For higher $\mathrm{Ga}$ concentrations the contact angle is expected to be larger.

For nanowires with an estimated Ga concentration lower than 0.50 the condition for TPB nucleation $(\Delta \gamma<0)$ is fulfilled for both side facet orientations for contact angles close

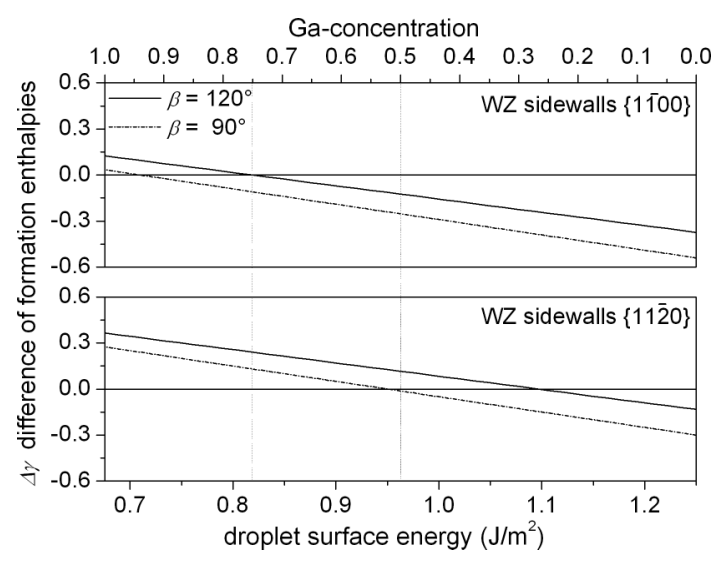

FIG. 6. Condition for TPB nucleation: $\Delta \gamma$, the difference of formation enthalpy for $\mathrm{C}$ and TPB nucleation, is plotted as a function of the liquid surface energy (lower $x$ axis) and the Ga concentration (upper $x$ axis) of the catalyst droplet. Two orientations of the WZ sidewalls $1 \overline{1} 00$ (upper graph) and $11 \overline{2} 0$ (lower graph) and two contact angles $\beta=90^{\circ}$ (dashed line) and $\beta=120^{\circ}$ (solid line) are considered. For $\Delta \gamma<0$ nucleation at the TPB takes place and the formation of the WZ crystal structure is favored. to $90^{\circ}$. The theory predicts that the formation of nanowires with WZ crystal structure is energetically favored for certain ranges of the relevant interface energies. This is in agreement with our observations where nanowires with a NWD ratio $<0.1$ show pure WZ crystal structure (see Fig. 4).

For nanowires with Ga concentration $>0.75$ (NWD $\geqslant 0.4$ ) and $\{11 \overline{2} 0\}$ oriented side facets the condition for nucleation at the TPB is not fulfilled and the nucleation at the center position is energetically favored. As a consequence nanowires with $\mathrm{ZB}$ crystal structure should form. For such high Ga concentrations during nanowire growth the growth conditions are very similar to the self-catalyzed $\mathrm{Ga}$ assisted growth mode where the catalyst droplet consists of $100 \% \mathrm{Ga}$. For the $\mathrm{Ga}$ assisted nanowire growth it is reported that nucleation at the center position occurs and pure ZB nanowires can be obtained. , $^{96,29}$ In addition, however, a dependence of the crystal structure on the $\mathrm{As}_{4}$ flux is observed: Depending on the $\mathrm{As}_{4} \mathrm{BEP}$ value pure $\mathrm{ZB}$ nanowires or nanowires with $\mathrm{ZB}$ and $\mathrm{WZ}$ segments are formed. It is assumed that the reason for this observation is an influence of the $\mathrm{As}_{4}$ pressure on the surface reconstruction. ${ }^{9}$

The reason why we observe only $\mathrm{ZB}$ segments within a $\mathrm{WZ}$ crystal structure matrix and not $\mathrm{ZB}$ as the dominant crystal structure is most likely due to the chosen $\mathrm{As}_{4}$ flux. If we compare our growth parameters with the data given in Ref. 9 for $\mathrm{Ga}$ assisted nanowire growth, we are indeed in a growth regime where a mixed crystal structure is likely to appear.

Our findings indicate that the catalyst droplet can accumulate $\mathrm{Ga}$ over a threshold value, where the growth can be considered as pseudo-Ga assisted and no longer typical $\mathrm{Au}$ catalyzed.

\section{CONCLUSION}

We study the correlation between the Ga concentration of the catalyst droplet during growth and the observed crystal structure for Au catalyzed GaAs nanowires. By a postgrowth analysis of the catalyst droplet using HRTEM and EDX we estimate the Ga concentration of the catalyst droplet during growth. We investigate five different samples which mainly differ in the chosen $\mathrm{As}_{4} / \mathrm{Ga}$ flux ratio. Depending on the Ga concentration of the catalyst droplet during growth we observe two growth regimes. At low Ga concentrations of 25-50 at. \% typical Au catalyzed growth is obtained. The dominant crystal structure is WZ. At Ga concentrations higher than 75 at. \% a new growth mode is observed which we refer to as pseudo-Ga-assisted growth resulting in a mixed $\mathrm{WZ}+\mathrm{ZB}$ crystal structure. The enhanced probability to form $\mathrm{ZB}$ segments is assigned to the reduced droplet surface energy of the catalyst resulting in nucleation at the center of the nanowire-catalyst boundary. Provided that in particular the $\mathrm{As}_{4} / \mathrm{Ga}$ ratio is adjusted, it should be possible to fully exploit the potential of the pseudo-Ga-assisted growth technique to form pure stacking fault free ZB nanowires. Indeed, investigated nanowires of sample E-generated at Ga rich growth conditions - already showed ZB as the dominant crystal structure, however with a high density of stacking faults. Further optimization of the growth parameters is currently being carried out. 


\section{ACKNOWLEDGMENTS}

We thank D. Weiss for access to the clean room and the chemical laboratory and T. Wojtowicz, A. Petroutchik, and L. T. Baczewski for the preparation of the Au covered sub- strates for nanowire growth. Our work was supported by Grant No. SFB 689, "Spin Phenomena in Reduced Dimensions." E. R. acknowledges financial help from the European Research Associates Nanoscience Project QOptInt.
${ }^{1}$ C. Thelander, T. Mårtensson, M. T. Björk, B. J. Ohlsson, M. W. Larsson, L. R. Wallenberg, and L. Samuelson, Appl. Phys. Lett. 83, 2052 (2003).

${ }^{2}$ C. Thelander, P. Agarwal, S. Brongersma, J. Eymery, L. F. Feiner, A. Forchel, M. Scheffler, W. Riess, B. J. Ohlsson, U. Gösele, and L. Samuelson, Materials Today 9, 28 (2006).

${ }^{3}$ T. Bryllert, L.-E. Wernersson, T. Löwgren, and L. Samuelson, Nanotechnology 17, 227 (2006).

${ }^{4}$ J. A. Czaban, D. A. Thompson, and R. R. LaPierre, Nano Lett. 9, 148 (2009).

${ }^{5}$ E. D. Minot, F. Kelkensberg, M. v. Kouwen, J. A. van Dam, L. P. Kouwenhoven, V. Zwiller, M. T. Borgström, O. Wunnicke, M. A. Verheijen, and E. P. A. M. Bakkers, Nano Lett. 7, 367 (2007).

${ }^{6}$ J. Heinrich, A. Huggenberger, T. Heindel, S. Reitzenstein, S. Hfling, L. Worschech, and A. Forchel, Appl. Phys. Lett. 96, 211117 (2010).

${ }^{7}$ S. Breuer, C. Pfüller, T. Flissikowski, O. Brandt, H. T. Grahn, L. Geelhaar, and H. Riechert, Nano Lett. 11, 1276 (2011).

${ }^{8}$ B. P. Timko, T. Cohen-Karni, Q. Qing, B. Tian, and C. M. Lieber, IEEE Transaction on Nanotechnology 9, 269 (2010).

${ }^{9}$ D. Spirkoska, J. Arbiol, A. Gustafsson, S. Conesa-Boj, F. Glas, I. Zardo, M. Heigoldt, M. H. Gass, A. L. Bleloch, S. Estrade, M. Kaniber, J. Rossler, F. Peiro, J. R. Morante, G. Abstreiter, L. Samuelson, and A. Fontcuberta i Morral, Phys. Rev. B 80, 245325 (2009).

${ }^{10}$ A. Fontcuberta i Morral, C. Colombo, J. Arbiol, J. R. Morante, and G. Abstreiter, Appl. Phys. Lett. 92, 063112 (2008).

${ }^{11}$ J. C. Harmand, G. Patriarche, N. Pr-Laperne, M.-N. Mrat-Combes, L. Travers, and F. Glas, Appl. Phys. Lett. 87, 203101 (2005).

${ }^{12}$ K. Dick, Progress in Crystal Growth and Characterization of Materials 54, 138 (2008).

${ }^{13}$ M. C. Plante and R. R. LaPierre, J. Cryst. Growth 286, 394 (2006).

${ }^{14}$ M. C. Plante, J. Garrett, S. C. Ghosh, P. Kruse, H. S. T. Hall, and R. R. LaPierre, Appl. Surf. Sci. 253, 2348 (2006).

${ }^{15}$ V. G. Dubrovskii, N. V. Sibirev, G. E. Cirlin, J. C. Harmand, and V. M.Ustinov, Phys. Rev. E 73, 021603 (2006).
${ }^{16}$ F. Glas, J. C. Harmand, and G. Patriarche, Phys. Rev. Lett. 99, 146101 (2007).

${ }^{17}$ T. B. Joyce and T. J. Bullough, J. Cryst. Growth 127, 265 (1993).

${ }^{18}$ M. C. Plante and R. R. LaPierre, Nanotechnology 19, 495603 (2008).

${ }^{19}$ M. Tchernycheva, J. C. Harmand, G. Patriarche, L. Travers, and G. E. Cirlin, Nanotechnology 17, 4025 (2006).

${ }^{20}$ For in particular $\mathrm{Ga}$ rich growth conditions the diameter of the catalyst droplet is larger than the diameter of the nanowire, resulting in a contact angle which is larger than $90^{\circ}$ (see Ref. 16).

${ }^{21}$ V. G. Dubrovskii, N. V. Sibirev, J. C. Harmand, and F. Glas, Phys. Rev. B 78, 235301 (2008).

${ }^{22}$ A. I. Persson, M. W. Larsson, S. Stenström, B. J. Ohlsson, L. Samuelson, and L. R. Wallenberg, Nat. Mater. 3, 677 (2004).

${ }^{23}$ R. Elliot and F. A. Shunk, Bull. Alloy Phase Diagrams 2, 356 (1981).

${ }^{24}$ C. Chatillon, F. Hodaj, and A. Pisch, J. Cryst. Growth 311, 3598 (2009).

${ }^{25}$ As EDX measurements showed a considerably lower $x_{\mathrm{Ga}}$ in the postgrowth catalyst droplet for nanowires with a NWD ratio $>0.3$ compared to nanowires with a NWD ratio $<0.1$ (see Fig. 2), and as theoretical values for $x_{\mathrm{As}}$ are very low, these assumptions are well justified.

${ }^{26}$ G. E. Cirlin, V. G. Dubrovskii, Y. B. Samsonenko, A. D. Bouravleuv, K. Durose, Y. Y. Proskuryakov, B. Mendes, L. Bowen, M. A. Kaliteevski, R. A. Abram, and D. Zeze, Phys. Rev. B 82, 035302 (2010).

${ }^{27}$ N. V. Sibirev, M. A. Timofeeva, A. D. Bolshakov, M. V. Nazarenko, and V. G. Dubrovskii, Phys. Solid State 52, 1531 (2010).

${ }^{28} \gamma_{\mathrm{LV}}$ of pure gold and pure gallium are calculated for a growth temperature of $540{ }^{\circ} \mathrm{C}$ using the linear interpolation formula $\gamma_{\mathrm{LV}}(T)=\gamma_{\mathrm{LV}}\left(T_{m}\right)-c\left(T-T_{m}\right)$ with $T_{m}$ as the melting temperature of the element and $c$ as an element specific coefficient $(c=$ $1.0 \times 10^{-4} \mathrm{~J} / \mathrm{m}^{2}{ }^{\circ} \mathrm{C}$ for $\mathrm{Au}$ and $7.0 \times 10^{-5} \mathrm{~J} / \mathrm{m}^{2}{ }^{\circ} \mathrm{C}$ for $\mathrm{Ga}$ ) as stated in Ref. 26. The surface energy of pure liquid $\mathrm{Au}(\mathrm{Ga})$ equals $1.145 \mathrm{~J} / \mathrm{m}^{2}\left(0.711 \mathrm{~J} / \mathrm{m}^{2}\right)$ at $T_{m}=1065^{\circ} \mathrm{C}\left(30^{\circ} \mathrm{C}\right)$.

${ }^{29} \mathrm{P}$. Krogstrup, R. Popovitz-Biro, E. Johnson, M. H. Madsen, J. Nygard, and H. Shtrikman, Nano Lett. 10, 4475 (2010). 